\title{
CÓMO ACTUALIZAR LA DEUDA PÚBLICA INTERNA OLVIDADA POR EL GOBIERNO PERUANO
}

\author{
HOW TO UPDATE THE INTERNAL PUBLIC DEBT FORGOTTEN BY \\ THE GOVERNMENT IN PERU \\ Esteban Marino Avelino SÁnchez * \\ Docente Asociado de la Facultad de Ciencias Contables \\ Universidad Nacional Mayor de San Marcos-UNMSM / Lima-Perú \\ [Recepción: Setiembre de 2014/ Conformidad: Octubre 2014]
}

\section{RESUMEN}

¿Cómo actualizar la deuda de los Bonos de Reconstrucción y Bonos de Desarrollo emitidos por el Gobierno peruano en monedas que se retiraron de circulación?, constituye el problema que en este artículo tratamos de explicar. La operación actualizar requiere, previamente, discernir sobre el bien económico o indicadores referentes de valor que se aplicarán en las operaciones de cálculo; en ese sentido, proponemos dos procedimientos: la actualización con el dólar estadounidense, dolarizando la deuda en la fecha de emisión de los bonos, más intereses, y la actualización de la deuda con IPC; con los cuales resultan sumas razonables que; sin embargo, desvanecen las expectativas de los tenedores de los bonos de reconstrucción y bonos de desarrollo, ilusionados por valores desmesurados de los mismos activos financieros de poca cuantía, expuestos a la hiperinflacionario; algunos creen que su poder adquisitivo aumentó en decenas de millones, cuando en realidad la hiperinflación tiene efectos de sentido contrario.

\section{Palabras clave:}

Deuda pública interna olvidada; bonos de reconstrucción; bonos de desarrollo; actualización de bonos; sobrevaloración de la deuda.

\begin{abstract}
How to update the debt of the Bonds for Reconstruction and Development Bonds issued by the Peruvian Government in currencies that were withdrawn from circulation?

Constitutes the problem that this article trying to explain. The operation requires updating previously discerning about the good of economic and the indicators for value to be applied in the calculations; in that sense, we propose two methods: Update the US dollar, dollarized debt at the date of issuance of the bonds, plus interest, and updating debt IPC; with which it sums are reasonable; however, expectations fade bondholders reconstruction and development bonds, excited by outsized financial same active low amount of values exposed to hyperinflation; some believe that their purchasing power increased by tens of millions, when in fact hyperinflation has effects opposite direction.
\end{abstract}

\section{Keywords:}

Forgot the domestic public debt; reconstruction bonds; development bonds; Update bonds; overvaluation of debt.

\footnotetext{
* Magíster en Contabilidad con mención en Auditoría. Contador Público y Economista Colegiado. Email: estebanavelino@gmail.com ** Colaborador: Jaime Bernabé Torres Ramírez. Docente Contratado de la Facultad de Ciencias Contables.

*** Colaborador: Richard Tipe Torvisco. Docente Contratado de la Facultad de Ciencias Contables.
} 


\section{INTRODUCCIÓN}

El tema actualización del valor de los Bonos de Reconstrucción (como de Desarrollo), aún pendientes de pago, lo elegimos como tema de este artículo porque algunos "peritos", por falta de metodología apropiada vinculante, sobrevaloran del valor actualizado de los bonos mencionados, con lo cual generan falsas expectativas a sus clientes. Esas sobrevaloraciones requieren análisis y propuestas de actualización de deudas en monedas, hoy, fuera de circulación; que conduzcan a resultados razonables. La sobrevaloración de deudas en moneda nacional, luego de la hiperinflación, genera falsas expectativas y litigios judiciales, lo que requiere explicación a fin de coadyuvar a la solución de los problemas de actualización de deudas en monedas fuera de circulación, por ello, es importante el tema elegido.

El objetivo de este artículo es, por lo tanto, presentar dos alternativas de actualización de deudas, cuyo signo monetario se retiró de circulación (soles oro e intis), con el dólar estadounidense e IPC. Exponemos, seguidamente: el marco conceptual, la metodología, el marco legal de los bonos de reconstrucción y de desarrollo, las falsas expectativas por "errores" de cálculo, la actualización de la deuda con el dólar estadounidense, la actualización de la deuda, con el IPC y las conclusiones.

\section{MARCO CONCEPTUAL}

El valor de cambio del dinero (en bonos) respecto de otras mercancías cambia constantemente. En general, después de algún tiempo con la misma cantidad de dinero se compra menos bienes; ciertamente, una unidad monetaria en términos nominales es la misma. Sin embargo, su valor de cambio disminuye; su valor económico a través del tiempo difiere de su valor nominal, lo que sustenta el principio financiero: un dólar del día de hoy vale más que un dólar de mañana. En efecto, si usted tiene S/. 1000 hoy lo puede invertir y ganar intereses, cosa que no puede hacer con S/. 1000 por recibirlo dentro de un año.

En los textos de finanzas abundan ejemplos del valor actual de inversiones por recibir en el futuro; en sentido contrario, poco. En situaciones de estabi- lidad económica, con inflación anual por debajo de dos dígitos, las deudas vencidas de cualquier plazo se pueden actualizar con facilidad; en situaciones hiperinflacionarias, donde los signos monetarios cambian en poco tiempo, la actualización de obligaciones vencidas hace muchos años generan controversias: pueden estar sobrevaloradas o subestimadas, ¿cómo saberlo?, he ahí el problema.

El artículo $1235^{\circ}$ del Código Civil, faculta a las partes la actualización del monto de una deuda contraída en moneda nacional con el índice de reajuste automático que fije el BCRP (tal índice consiste en acumular diariamente la inflación del mes anterior), con otras monedas o mercancías, a fin de mantener dicho monto en valor constante.

Si utiliza el índice de reajuste automático o el IPC mantiene el valor constante de la deuda en el mercado interno, respecto a la canasta de bienes y servicios de consumo final; si utiliza el dólar estadounidense mantiene el valor constante de la deuda, respecto al dólar.

Las señales del mercado de valores constituyen argumentos objetivos de los cambios de valor del activo financiero objeto de valoración, facilitan elegir el indicador económico referente de valor, que aplicado a la actualización de deudas permite obtener resultados razonables.

\section{METODOLOGÍA}

Descripción, concisa, del marco legal de los bonos de reconstrucción y bonos de desarrollo como de los problemas de su actualización.

Técnicas utilizadas: observación, cálculos y análisis de información procesada.

Los factores de interés y de actualización con el IPC fueron realizados en la hoja de cálculo y se utilizan con todos los decimales. Si verifica los cálculos con la información que aparece a la vista puede haber diferencias no significativas.

\section{MARCO LEGAL DE LOS BONOS DE RECONSTRUCCIÓN}

Ley No 23592 (promulgada 20/04/1983), Decretos Supremos Nos 178 y 210-83-EFC (El Peruano: 25/05/1983 y 05/06/1983, respectivamente). 
Por Ley No 23592, ley para contribuir a la atención, rehabilitación y construcción de la zonas afectadas por los fenómenos de la naturaleza ocurridos en 1983, se autoriza al Poder Ejecutivo a emitir Bonos de Reconstrucción en moneda nacional hasta por 200 mil millones de soles oro, en dos series: A y B. Los bonos de serie A, fueron de suscripción obligatoria en forma mensual desde mayo de 1983 hasta abril de 1984; por las persona naturales con ingresos mensuales superiores a 6 unidades de referencia mensual (D.S. No 001-83-TR): $10 \%$ de sus ingresos mensuales, retenido por los empleadores; por las personas jurídicas: un dozavo del $10 \%$ de sus reservas de libre disposición. Los bonos de serie $\mathrm{B}$ fueron de suscripción voluntaria (Artículos $\mathrm{No}_{\mathrm{s}} 3,5,6$ y $7^{\circ} \mathrm{de}$ la Ley $\mathrm{N}^{\circ} \mathrm{s} 23592$ ).

El Artículo 30 del Decreto Supremo No 178-83EFC faculta a la Dirección General de Tesoro Público a emitir los bonos indicados, cuyas características serán determinadas por decreto supremo. La serie A dividida en 12 sub series, con vencimiento mensual; el 30 de cada mes, a partir de mayo de 1985 hasta abril de 1986. La serie B, con vencimiento a los dos años de su colocación la que deberá ocurrir entre mayo de 1983 y abril de 1984. Los valores nominales de los bonos de reconstrucción serán múltiplos de 1000, de 1 hasta 100 mil soles oro, al 56\% capitalizable trimestralmente, pagaderos en la fecha de redención. A partir de 1985, se consignará en los presupuestos del sector público nacional las partidas necesarias para atender los servicios de los bonos de reconstrucción

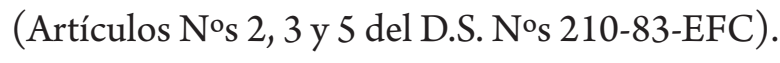

¿Cuánto fue el poder de compra de un bono de reconstrucción de 100 mil soles oro en la fecha de emisión, ejemplo 30 de setiembre de 1983? Aplicando el tipo de cambio de la misma fecha (S/.2 052.00 por US \$1.00) sólo US \$48.73; de un bono de 1000 soles oro, de la misma fecha, solo US $\$ 0.49$. Es decir, sumas no significativas que, por el simple transcurso del tiempo: 25 a 30 años, no pueden hacer millonarios a sus tenedores.

\section{MARCO LEGAL DE LOS BONOS DE DESARROLLO}

Ley No 24030 (promulgada: 14/12/1984), Decreto Supremo No 035-85-EF (El Peruano: 05/02/1985).

Por Ley No 24030, ley de normas relativas al financiamiento del sector público, se crearon los Bonos de Desarrollo de suscripción obligatoria y, se autorizó al Poder Ejecutivo a emitirlos durante el año 1985, destinados al financiamiento de mejoras socioeconómicas de las zonas deprimidas, declaradas en emergencia, cuyas condiciones específicas se fijarán por Decreto Supremo. Las personas naturales y jurídicas fueron obligadas a suscribir los bonos de desarrollo, de acuerdo con lo establecido en la Ley No 23592 y disposiciones complementarias y reglamentarias para los bonos de la serie $\mathrm{A}$, las que tuvieron plena vigencia durante 1985 (artículo 10 de la Ley $\mathrm{N}^{\circ} 24030$ ).

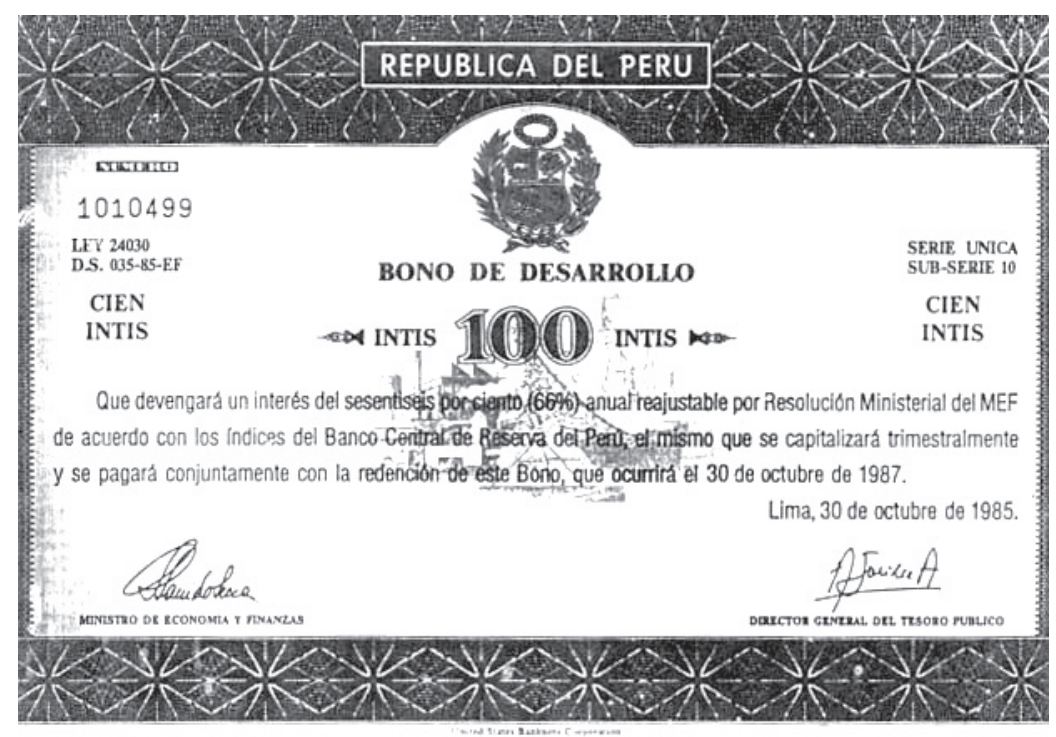

Vol. 22(42) 2014 | QVIPURAMAYOC /63 
Por Decreto Supremo No 035-85-EF se dispone la emisión de los bonos de desarrollo hasta por la suma de 428 mil millones de soles oro en serie única, dividida en doce sub-series, con vencimiento mensual; el 30 de cada mes, de enero a diciembre de 1987. Los valores nominales de los bonos de desarrollo serán múltiplos de 5000 , de 5 hasta 200 mil soles oro, al $66 \%$ anual capitalizable semestralmente, pagaderos en las fechas de redención (Artículos del $1^{\circ}$ al $5^{\circ}$ del D.S. 035-85-EF) ¿Cuánto fue el poder de compra de un bono de desarrollo de 200 mil soles oro en la fecha de emisión, ejemplo 30 de octubre de 1985 ? Aplicando el tipo de cambio (S/.17 511.00 por US \$1.00) de la misma fecha, sólo US \$11.42; de un bono de 5 mil soles oro de la misma fecha, solo US \$ 0.30. Es decir, sumas no significativas que, por el simple transcurso del tiempo: 25 a 30 años, no pueden hacer millonarios a sus tenedores.
Debido a su poca cuantía, miles de bonos de reconstrucción y bonos de desarrollo no se habrían pagado, lo que constituye una parte de la deuda pública interna olvidada.

\section{FALSAS EXPECTATIVAS POR “ERRORES” DE CÁlCULO}

Algunos estudios de abogados tienen fajos de bonos de reconstrucción y bonos de desarrollo, algunos actualizados con sumas millonarias, lo que despierta falsas expectativas a sus tenedores. Tengo a la vista la actualización, en tres páginas ( 4 cuadros), de un bono de reconstrucción de 50 mil soles oro con tasa de interés $56 \%$ anual capitalizable trimestralmente, emitido el 30 de setiembre de 1983 y fecha de redención dos años después, cuya actualización se resume en la tabla No 1 .

Tabla No 1

Actualización bonos con artificios ilícitos

\begin{tabular}{|c|l|l|}
\hline Cuadro & \multicolumn{1}{|c|}{ Operaciones } & \multicolumn{1}{|c|}{ Valor actualizado } \\
\hline 1 & $50000(1.14)^{8}$ & $\mathrm{~S} / .142,629.32(\mathrm{I} / .142 .63) ; 1 /$ \\
\hline 2 & $142.63 \times 3594029.952 ; 2 /$ & $\mathrm{I} / 512614052.07(\mathrm{~S} / .512 .61) ; 2 /$ \\
\hline 3 & $512.61 \times 233323.8712 \times 1.424517 ; 3 /$ & $\mathrm{S} / .170378144 .40 ; 3 /$ \\
\hline 4 & $\mathrm{~S} / .170378144 .40 \times 1.0914773 ; 4 /$ & $\mathrm{S} / .185963876 .21 ; 4 /$ \\
\hline
\end{tabular}

1/ Deuda actualizada con la tasa de interés establecida en el bono a la fecha de redención: al 30 de setiembre de 1985, S/. 142,629.32 (soles oro), su equivalente 142.63 intis.

2/ Multiplicando la deuda en intis por 3594029.952 (factor de actualización obtenido del IPC, indebidamente desde enero de 1985, hasta el 30 de octubre de 2005) resulta 512.61 millones de intis; su equivalente en Nuevos Soles 512.61.

3/ Multiplicando 512.61 por 233323.8712 (factor de actualización de interés legal), luego este resultado por 1.424517 (artificio del "experto" que efectuó el cálculo), resulta 170 millones de nuevos soles al 31 de marzo de 2007 , lo cual es absurdo.

4/ Multiplicando 170 (considerando todos los dígitos) por 1.0914773 (factor de la tasa de interés legal, del periodo abril de 2007 al 31 de diciembre de 2009), resulta 185.9 millones de nuevos soles, absurdo.

* Elaboración propia.

En el periodo octubre de 1985 a octubre de 2005 se hace doble actualización: una con la inflación anual, con la cual resulta S/. 512.63 (nuevos soles), y otra, con interés legal sobre esta suma actualizada; además, de un artificio para elevar el valor actualizado a poco más de 170 millones de nuevos soles.

\section{ACTUALIZACIÓN RAZONABLE CON EL DÓLAR ESTADOUNIDENSE}

Valor nominal del Bono de Reconstrucción: S/. 50000.00 (soles oro) de setiembre de 1983.

Su poder de compra, respecto al dólar. Solo US \$ 24.37. Sin embargo, esta suma es tres veces más el valor del bono actualizado con la tasa de 56\% 
capitalizable trimestralmente, como se demuestra en la tabla No 2. La conversión de la deuda en la fecha de origen, a dólares mantiene su valor constante respecto al dólar.

Tabla No 2

Actualización del bono de reconstrucción con el dólar estadounidense

\begin{tabular}{|c|c|c|c|}
\hline Fecha & Deuda, S/. & Tipo cambio $^{*}$ & Deuda, US \$ \\
\hline $30 / 09 / 1983$ & $50,000.00$ & $2,052.00$ & 24.37 \\
\hline $30 / 09 / 1985$ & $142,629.32$ & $17,468.00$ & 8.17 \\
\hline
\end{tabular}

* Fuente: SBS. Documento: Tipos de cambio de 1930 a 1999

Adicionalmente, puede agregar intereses. Aplicando a la deuda en dólares $7 \%$ anual, la tasa de los bonos del tesoro del Gobierno de Estados Unidos señalada por los expertos o alternativamente (sólo como curiosidad) el interés legal conforme a la legislación peruana.

Primera opción, tasa: $7 \%$ nominal anual.

Periodo de intereses: del30/09/1985 al30/09/2014: 11323 días.

Deuda actualizada $=24.37(1.07) 11323 / 360=$ US $\$ 204.63$

La tasa promedio de los bonos del tesoro de EE UU podría ser menor, la capitalización de intereses discutible. Sin embargo, US \$ 204.63 (al tipo de cambio S/. 2.93 por dólar, S/.600.00), el valor constante de la deuda más intereses, constituye una referencia razonable, un límite a la sobrevaloración.

Segunda opción, con la tasa de interés legal, conforme a la legislación peruana, la deuda actualizada a la misma fecha es menor. El factor acumulado de las diversas tasas de interés legal desde el 30/09/1985 hasta el 30/09/2014 es 5.722743; multiplicando este factor por 24.37 resulta solo US \$139.46.

Valor nominal del Bono de Desarrollo: I/. 100.00 de octubre de 1985.

$\mathrm{Su}$ poder de compra, respecto al dólar fue US \$ 5.71; adicionando intereses calculados con la tasa $7 \%$ anual resulta solo US \$ 41.34 (al tipo de cambio S/. 2.93 por dólar, S/. 121.13).

\section{Tabla No 3}

Actualización del bono de desarrollo con el dólar estadounidense

\begin{tabular}{|c|c|c|c|}
\hline Fecha & Deuda, S/. & Tipo cambio $^{*}$ & Deuda, US \$ \\
\hline $30 / 10 / 1985$ & 100.00 & 17.51 & 5.71 \\
\hline Interés & & & \\
\hline Del & $30 / 10 / 1985$ & Deuda actualizada & 41.34 \\
\hline $\mathrm{Al}$ & $30 / 09 / 2014$ & Tasa & \\
\hline Días & 10562 & $7 \%$ & \\
\hline
\end{tabular}

* Fuente: SBS. Documento: Tipos de cambio de 1930 a 1999

La dolarización de la deuda más intereses calculados con la tasa $7 \%$ anual da resultados razonables.

\section{ACTUALIZACIÓN CON EL IPC}

Valor nominal del Bono de Reconstrucción: S/. 50000.00 (soles oro) de setiembre de 1983.

Aplicando el IPC a la deuda desde la fecha de origen, setiembre de 1983 hasta agosto de 2014, tampoco resulta un monto elevado, como lo puede comprobar en la tabla No 4.

En la segunda línea de la parte superior se consigna la deuda de la fecha de origen, en soles oro y su equivalencia en nuevos soles (sólo para efectuar los cálculos). En la primera columna de la izquierda el año base del IPC, en la segunda columna la fecha (último día del mes y año indicado). La abreviatura 
FAC significa factor de actualización por periodo y total. Dividiendo 17.41973 entre 0.00002 resulta 887855.75943, multiplicando por 0.00005 resulta S/.44.39; dividiendo 100.127514 entre 11.083788 resulta 9.03369, multiplicando por 44.39 resulta S/. 401.03; los valores actualizados de los periodos siguientes se obtienen siguiendo el mismo procedimiento.

\section{Tabla No 4}

Actualización del bono de reconstrucción con el IPC

\begin{tabular}{|c|c|c|c|c|}
\hline & S/. (soles oro) & IPC & FAC & S/. (nuevos soles) \\
\hline Deuda * & $50,000.00$ & & & 0.00005 \\
\hline Año Base & Mes y año & & & \\
\hline \multirow[t]{2}{*}{1994} & Set. 1983 & 0.000020 & & \\
\hline & Dic. 1990 & 17.419730 & 887855.75943 & 44.39 \\
\hline \multirow[t]{2}{*}{ Dic. 2001} & Dic. 1990 & 11.083788 & & \\
\hline & Dic. 2000 & 100.127514 & 9.03369 & 401.03 \\
\hline \multirow[t]{2}{*}{2009} & Dic. 2000 & 81.629800 & & \\
\hline & Mar. 3007 & 90.652000 & 1.11053 & 445.35 \\
\hline \multirow[t]{3}{*}{2009} & Mar. 3007 & 90.652000 & & \\
\hline & Ago. 2014 & 115.927783 & 1.27882 & 569.53 \\
\hline & & Factor total & 11390595.69519 & \\
\hline
\end{tabular}

* En la fecha de emisión del bono

Al mismo resultado se llega multiplicando, 0.00005 x $11390595.69519=$ S/. 569.53.

Elaboración propia

Aunque la actualización dolarizando la deuda e intereses con la tasa de los bonos del tesoro del Gobierno de los Estados Unidos y la actualización con el IPC, son prácticamente los mismos, sugerimos la primera opción por la naturaleza del activo financiero objeto de actualización.
Valor nominal del Bono de Desarrollo: I/. 100.00 de octubre de 1985.

El valor actualizado: $0.0001 \times 1922107.91726=$ S/. 192.21, aunque es mayor al resultado mostrado en la tabla No 3 , esta suma tampoco es significativa.

Tabla No 5

Actualización del bono de desarrollo con el IPC

\begin{tabular}{|c|c|r|r|c|}
\hline & I/. (intis) & \multicolumn{1}{c|}{ IPC } & \multicolumn{1}{c|}{ FAC } & S/. (nuevos soles) \\
\hline Deuda $^{*}$ & 100.00 & & & 0.0001 \\
\hline Año Base & Mes y año & & & \\
\hline 1994 & Oct. 1985 & 0.000116 & & \\
\hline & Dic. 1990 & 17.419730 & 149821.36407 & 14.98 \\
\hline Dic. 2001 & Dic. 1990 & 11.083788 & & 135.34 \\
\hline & Dic. 2000 & 100.127514 & 9.03369 & \\
\hline 2009 & Dic. 2000 & 81.629800 & & 192.21 \\
\hline & Ago. 2014 & 115.927783 & 1.42016 & \\
\hline & & Factor total & 1922107.91726 & \\
\hline & Ago. 2014 & 115.927783 & 1.27882 & 569.53 \\
\hline
\end{tabular}

* En la fecha de emisión del bono Elaboración propia 


\section{CONCLUSIONES}

1. Los importes consignados en los bonos de reconstrucción y bonos de desarrollo fueron de poca cuantía. Por lo tanto, es ilusorio suponer que después de la hiperinflación de la década de los ochenta, hoy los mismos importes actualizados tengan poderes de compra desmesurados.

2. La actualización de la deuda del periodo: 1985 a 2005; primero con el IPC, luego esa deuda actualizada, se vuelve a actualizar con interés legal de enero de 1985 a octubre de 2007, el que incluye en parte la hiperinflación, es absurdo; peor todavía cuando el "experto" utiliza artificios para elevarlo exorbitantemente, revela que no entendió nada sobre el poder de compra de la deuda original.

3. La dolarización de la deuda más intereses, con la tasa de $7 \%$ anual da resultados razonables. Por la naturaleza de los activos financieros tratados, considerando los cambios de las tasas de interés de los bonos del tesoro de EE UU desde la fecha de emisión de los bonos hasta la actualidad, es recomendable para actualizar la deuda pública interna, en bonos, olvidada.

4. La actualización con el IPC, sin intereses ni artificios, también conduce a resultados razonables. Sin embargo para actualizar bonos no sería apropiado.
5. La hiperinflación no enriquece a la clase media, que fue obligada a comprar bonos en la década del ochenta, sino por el contrario, lo empobrece: todo el mundo lo sabe por experiencia propia o ajena.

\section{REFERENCIAS BIBLIOGRÁFICAS}

1. AVELinO E. (2011). Manual de Cálculo de Intereses. $6^{\circ}$ Edición. Lima - Perú. Editorial San Marcos.

2. AVELINO E. (2000). Manual de Cálculo de Intereses, tomo II. $3^{\circ}$ Edición. Lima - Perú. Editorial San Marcos.

3. Ley 23592 (promulgada: 20/04/1983).

4. Decreto Supremo 178-83-EFC (El Peruano: 25/05/1983).

5. Decreto Supremo 210-83-EFC (El Peruano: 05/06/1983).

6. Ley 24030 (promulgada: 14/12/1984).

7. Decreto Supremo 035-85-EF (El Peruano: 05/02/1985).

8. SBS: en portal electrónico, Factores de tasas de interés legal. www.sbs.gob.pe

9. INEI: en portal electrónico, IPC. http://www. inei.gob.pe/estadisticas/indice-tematico/economia/ 